\title{
Tuberculous myocarditis presenting as a refractory ventricular tachycardia of biventricular origin
}

\author{
M.P. Gautam ${ }^{1}$, G. Sogunuru², G. Subramanyam ${ }^{3}$, R.C. Viswanath ${ }^{4}$ \\ ${ }^{1}$ DM Cardiology Resident, ${ }^{2}$ Associate Professor, ${ }^{3}$ Professor, Department of Cardiology, College of Medical Sciences, \\ Bharatpur, Chitwan, Nepal, ${ }^{4} \mathrm{MD}$ Resident, Seton Hall University, Trenton, NJ, USA
}

\begin{abstract}
Ventricular tachycardia (VT) is one of the difficult clinical problems for the physician. Its evaluation and treatment are complicated because of its life-threatening nature and urgent need of rapid management. Any process that creates myocardial scar tissue could be the substrate for ventricular tachycardia. The coronary artery disease is the most common cause of myocardial scar. The dilated cardiomyopathies, hypertrophic cardiomyopathy, right ventricular dysplasia, Chagas disease, sarcoidosis, myocarditis including tubercular and other chronic granulomatous conditions and surgical incisions in the ventricle also can create myocardial scar and can lead to ventricular tachycardia. Occasionally, the arrhythmia may be well-tolerated, but in most of the situations it is associated with grave, life-threatening hemodynamic compromise. Regardless of the arrhythmia mechanism, the severity of clinical symptoms and hemodynamic compromise determines the urgency with which VT must be treated. Rarely, patients present with repetitive runs of nonsustained or sustained VT despite the medical treatment and poorly respond to the conventional treatment. Such refractory VT may cause a tachycardia-induced cardiomyopathy in long run. In such cases, long-term management also include looking beyond the VT and work up for the possible and treatable cause of VT. Here we are presenting a case report of a young patient with tubercular myocarditis who has presented to us with recurrent ventricular tachycardia of both right bundle and left bundle branch block morphology and LV dysfunction. A review of literature has been carried out on causes and management of refractory VT.
\end{abstract}

Key words: Ventricular tachycardia, refractory VT, tubercular myocarditis.

\section{Introduction}

Ventricular tachycardia (VT) is protean in form, duration, clinical setting and prognosis. It refers to any rhythm arising distal to the bundle of His at the rate faster than 100 (or 120) beats per minute.

Correspondence: M.P. Gautam

E-mail: manipdgautam@yahoo.com
The rhythm may arise from working ventricular myocardium and/or the distal conduction system. VT may be reflected in symptoms such as syncope, palpitations, and dyspnea. It is often, but not always, associated with hemodynamic compromise, particularly if the left ventricle is impaired or the heart rate is especially fast. When sustained VT causes signs or symptoms of diminished perfusion, 
emergent treatment is necessary. With some exceptions, VT is associated with increased risk of sudden death. Approach to it not only depends on the emergency management to revert it to normal sinus rhythm either by DC cardio version or by pharmacotherapy, but also on identification of the etiology and to cure it if possible. Though most common and most important clinical setting is ischemic or scar related VTs of coronary artery disease due to atherosclerosis, nonischemic causes are also not very uncommon, especially in young individuals. Etiological aspects of acute myocarditis may vary depending on the age, predisposing factors and geographical location of the patient. In tropical countries like Nepal and India where tuberculosis is endemic, it may be one of the rare but potential causes of myocarditis. Tuberculous involvement of the heart has been described in the form of pericardial effusion, constrictive or effusive-constrictive pericarditis and rarely coronary vasculitis leading to coronary artery obstruction. As effective therapeutic strategies are available for extra pulmonary tuberculosis, early diagnosis and prompt treatment effectively results in cure of the disease.

\section{Case summary}

A 33 year old Asian male, without any significant past medical history presented to the Emergency Room with complaints on palpitation, breathlessness, sweating and abdominal discomfort of 4 days duration. He lost weight of 8 kilograms over past 3 months. He denied smoking, any drug abuse or high risk sexual behavior. No family history of heart disease or sudden cardiac death was present. He was dyspneic, tachypneic, with a pulse rate of 190 bpm; BP 100/60mm Hg. Examination revealed intermittent cannon waves in JVP, normally placed apex, right and left ventricular gallop sounds, basal rales of lungs. ECG showed VT that had northwest axis with right bundle branch block $(\mathrm{RBBB})$ pattern (Figure 1). Taking hypoxemia $(\mathrm{SaO} 2=80 \%)$ and hemodynamic deterioration into consideration he was DC cardioverted and was intubated. Even though he became hemodynamically stable with electrocardiography showing normal sinus rhythm after DC shock (Figure 2), VT was recurrent with a different morphology - right axis deviation with left bundle branch block (LBBB) pattern (Figure 3). Chest X-ray was normal and Echocardiogram showed normal sized chambers with trivial mitral regurgitation, mild tricuspid regurgitation (PA pressure $33 \mathrm{mmHg}$ ) and an ejection fraction of 30 $-35 \%$. His BNP was within normal limits. His CPK-MB was 29 IU/L, Troponin T $<0.01 \mathrm{mg} / \mathrm{ml}$. Ultrasound abdomen showed fatty liver. Other laboratory data are summarized in Table1.

Upon conservative management he had improved with ejection fraction of $45-50 \%$ but continued to have frequent ventricular premature complexes (VPCs) and non-sustained ventricular tachycardia (NSVT) and occasional sinus rhythm.

His serial ECGs showed persisting VT with northwest axis RBBB pattern or right axis deviation with LBBB pattern. Only rarely he had sinus rhythm (Figure 4). A working diagnosis of non ischemic VT was made and he was evaluated for the 
Table 1. Laboratory data

\begin{tabular}{ll}
\hline PARAMETER & VALUE \\
\hline Hemoglobin & $13.7 \mathrm{gm} \%$ \\
Total leukocyte count & $16,200 / \mathrm{cmm}$ \\
Platelet count & $296,000 / \mathrm{cmm}$ \\
ESR & $10 \mathrm{~mm}$ at the end of $1^{\text {st }}$ hour \\
Bleeding time & $2 \mathrm{~min}$ \\
Clotting time & $7.30 \mathrm{~min}$ \\
Serum creatinine & $1.13 \mathrm{mg} / \mathrm{dl}$ \\
Sodium & $139 \mathrm{~m} \mathrm{~mol} / \mathrm{L}$ \\
Potassium & $4.4 \mathrm{~m} \mathrm{~mol} / \mathrm{L}$ \\
Serum albumen & $5.8 \mathrm{gm} / \mathrm{dl}$ \\
Serum globulins & $2.1 \mathrm{gm} / \mathrm{dl}$ \\
Serum bilirubin & $1.32 \mathrm{mg} / \mathrm{dl}$ \\
SGOT & $30 \mathrm{IU} / \mathrm{L}$ \\
SGPT & $37 \mathrm{IU} / \mathrm{L}$ \\
Alkaline phosphatase & $46 \mathrm{IU} / \mathrm{L}$ \\
TSH & $1.90 \mathrm{micro} \mathrm{IU/ml}$ \\
Mantoux test & $20.7 \mathrm{U} / \mathrm{L}(\mathrm{normal} 8-52 \mathrm{U} / \mathrm{L})$ \\
Serum ACE levels & \\
\hline &
\end{tabular}

possibilities like myocarditis, arrhythmogenic right and left ventricular dysplasia, inflammatory or infiltrative cardiac diseases. Repeat CXR was normal and echocardiography showed normal sized chambers with ejection fraction of $45 \%$ and mild tricuspid regurgitation (Figure 5). Cardiac MRI revealed abnormal delayed enhancement in interventricular septum and anteroapical wall and nonnecrotic subcarinal lymph node enlargement.
CT scan of chest showed infiltrated lesions of both lungs with ground-glass appearance with the possibility of infiltrative disease or sarcoidosis and also enlarged nonnecrotic sub carinal lymph node. He was investigated for infiltrative myocardial diseases. Serum ACE levels were within normal limits. His Mantoux test was positive (16 x 16 $\mathrm{mm}$ induration). For the confirmation, he underwent CT guided biopsy of the lymph node 
M.P. Gautam et al. Tuberculous myocarditis presenting as a refractory ventricular tachycardia

which showed necrotizing granulomatous continued to have VTs of both morphologies with lymphadenitis probable of mycobacterial etiology. a heart rate of 140 to $150 \mathrm{bpm}$.

He was started on antituberculous treatment, but

Figure 1: Ventricular tachycardia having right bundle branch block (RBBB) morphology

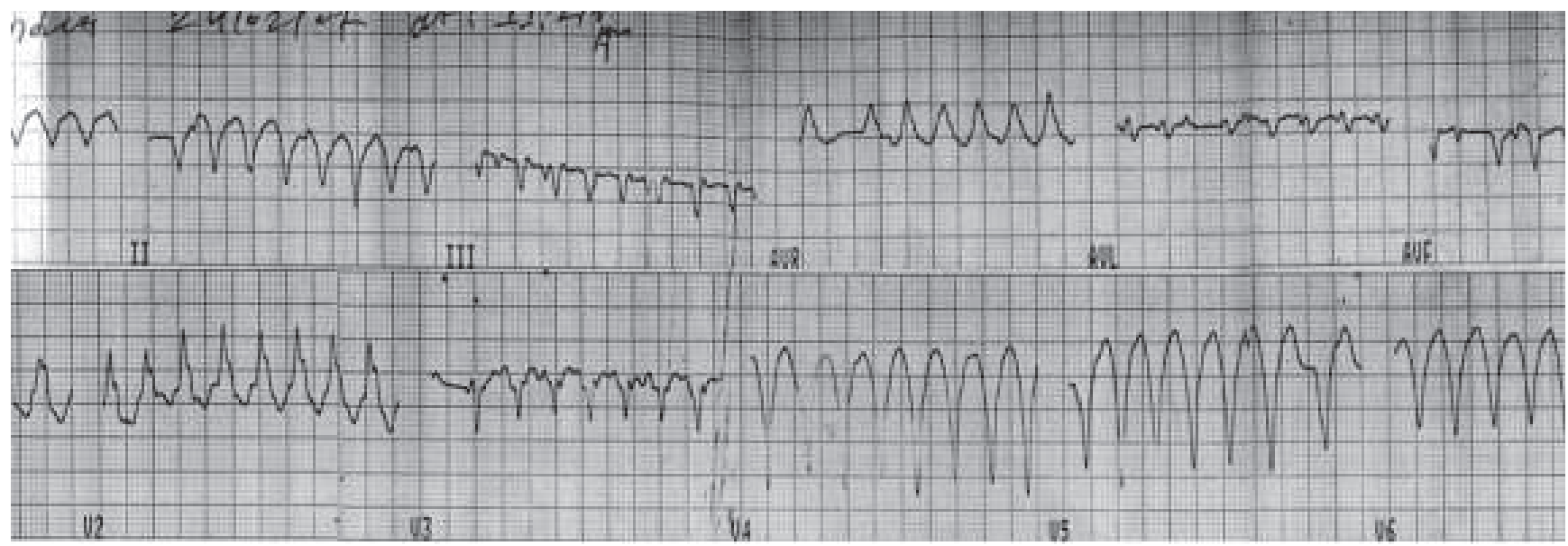

Figure 2: Sinus rhythm

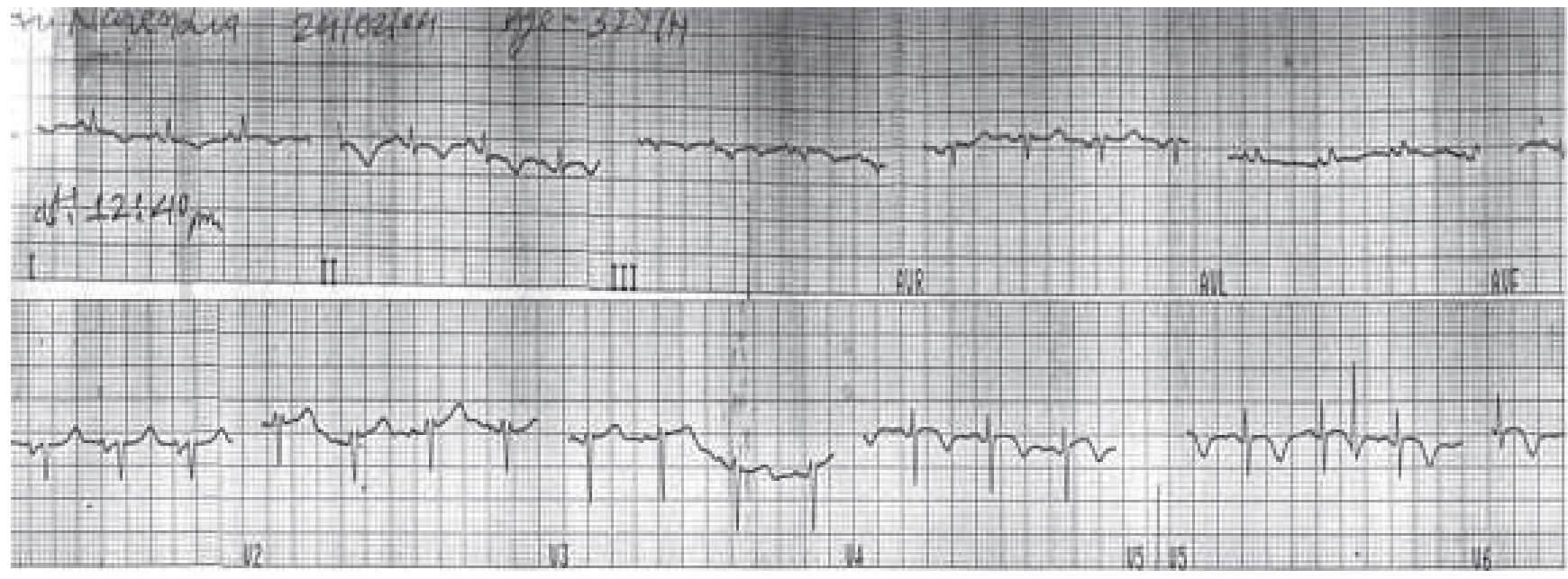

Figure 3. Ventricular tachycardia having left bundle branch block (LBBB) morphology

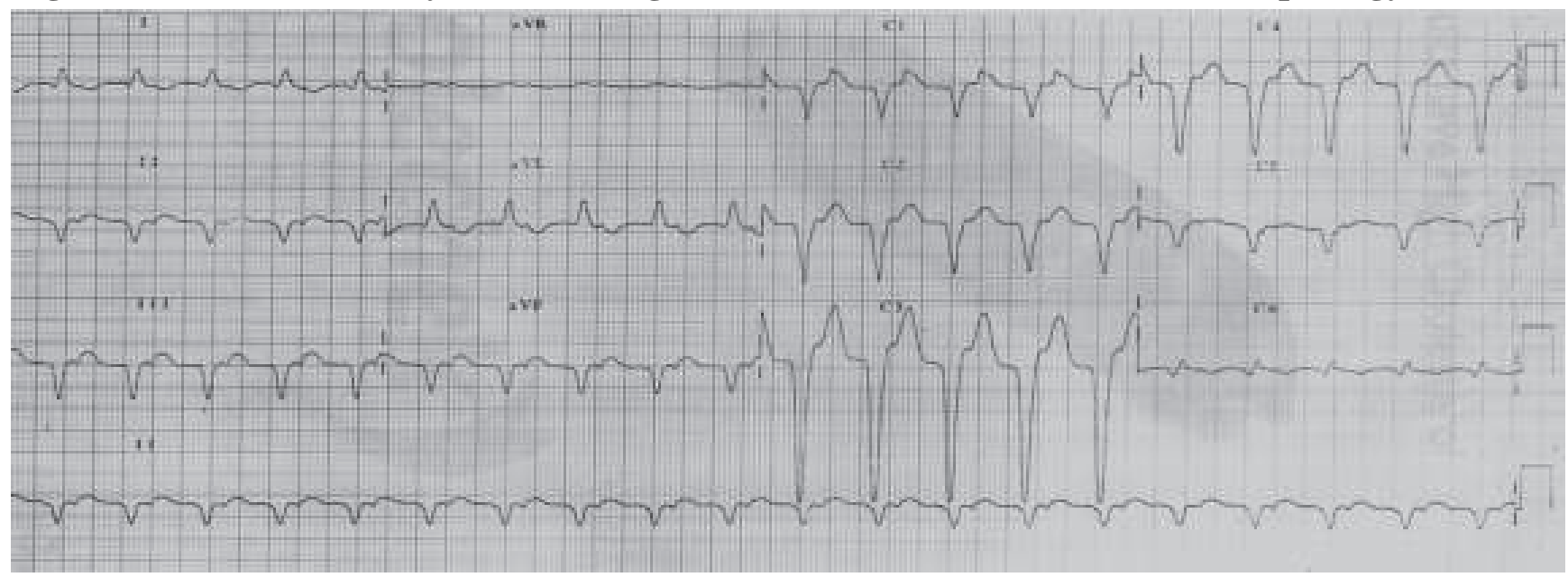


Journal of College of Medical Sciences-Nepal,2011, Vol-7,No-2

Fig - 4 Slow VT and occasional sinus rhythm

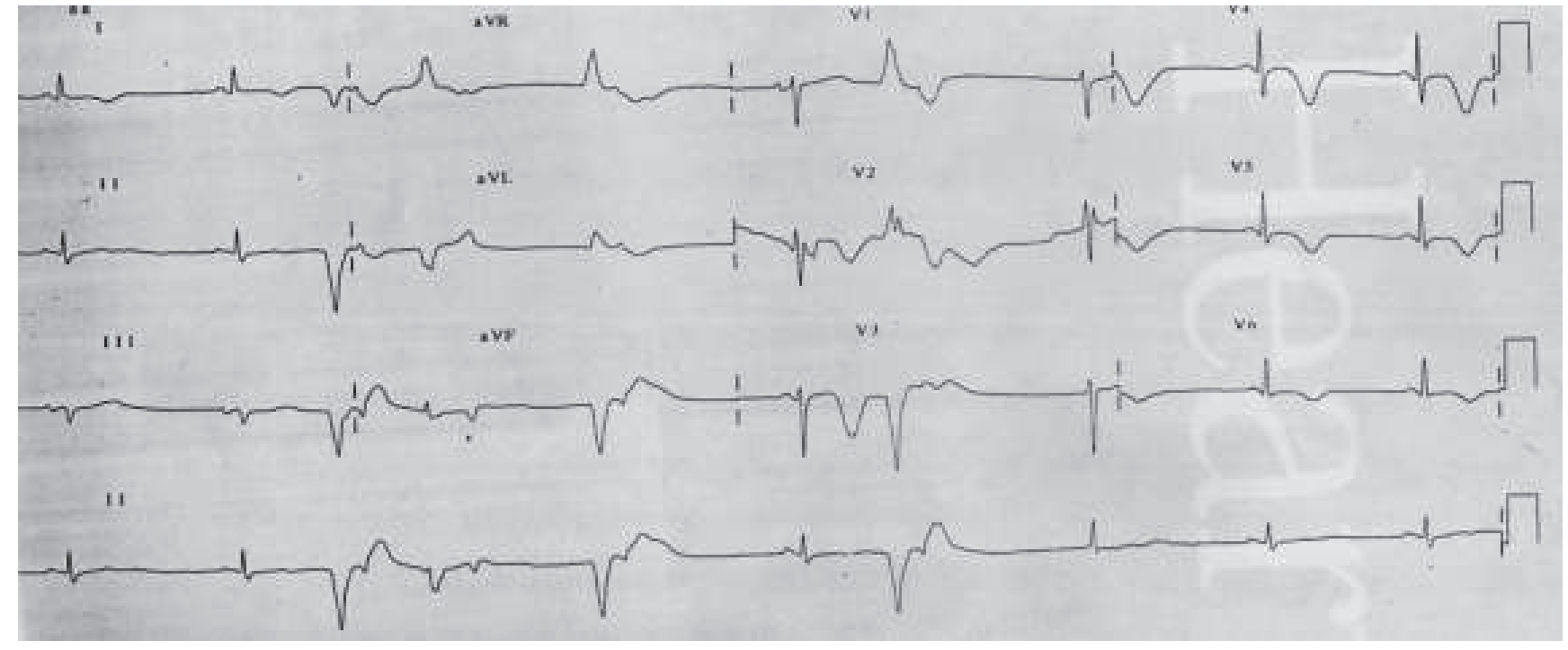

Figure 5. M mode echocardiography with assessment of LV systolic function.

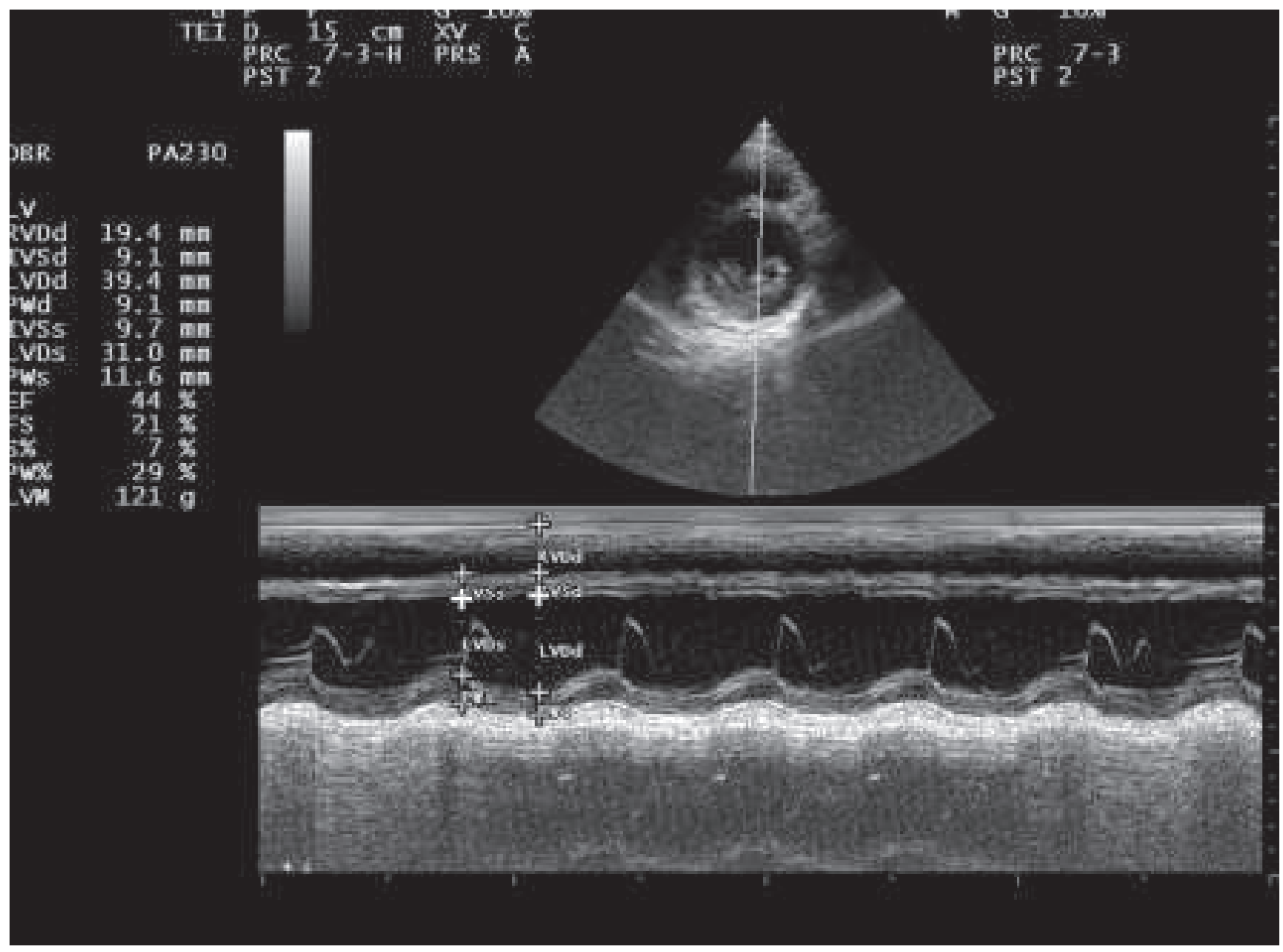


M.P. Gautam et al. Tuberculous myocarditis presenting as a refractory ventricular tachycardia

\section{Discussion}

Although tuberculosis primarily involves the lungs, $15-20 \%$ of all cases are extrapulmonary; the most common site being the lymphnodes, pleura, abdomen and central nervous system. ${ }^{1}$ Involvement of the heart (apart from the pericardium) is extremely rare and was first reported by Morgagni in 1761. Three distinct histological forms of myocardial tuberculosis are recognized: diffuse infiltrating (the most common form, characterized microscopically by giant cells and lymphocytes), milliary (resulting from hematogenous spread ) and nodular ( characterized by central caseation ). It has been described that cardiac tuberculomas can cause premature ventricular contractions arrhythmias, ${ }^{2}$ complete heart block, ${ }^{3,4}$ congestive heart failure, ${ }^{5}$ superior vena caval obstruction, ${ }^{6}$ right ventricular outflow tract obstruction, ${ }^{7}$ aortic insufficiency, ${ }^{8}$ and sudden cardiac death. ${ }^{9,10}$

Tuberculosis has been described as one of the rare causes of myocarditis. Myocarditis may present with rapidly progressive ventricular compromise or refractory ventricular arrhythmias. But the most common cause for this presentationis giant cell myocarditis, ${ }^{11}$ Vignola et al ${ }^{12}$ reported refractory ventricular tachycardia or aborted sudden cardiac death in 6 of 12 patients who underwent cardiac biopsy which demonstrated lymphocytic myocarditis. Recently two cases have been reported from India, presenting as ventricular arrhythmias, finally diagnosed to be of tuberculous involvement of the heart.

In any patient who presents with nonischemic ventricular arrhythmias all potentially treatable causes must be evaluated. Tuberculosis being endemic in Nepal high suspicion is needed to diagnose tuberculous myocarditis if all other causes of myocarditis have been ruled out. Especially noninvasive investigative modalities like cardiac MRI or CT scan chest will help to identify the tuberculous involvement of the heart or lungs. Identifying the underlying cause and mechanism of VT is crucial in terms of their management, more so for poorly responsive VT as such cases warrants for special attention and management. The management of patients with recurrent drug refractory ventricular tachycardia is difficult. Both conventional anti-arrhythmic drugs and antitachycardia pacing often provide only partial control, and some patients with implantable cardioverter defibrillators may be subjected to frequent and distressing shocks. Radiofrequency ablation, the mainstay of treatment is technically challenging, restricted to specific centers, and associated with significant procedural risks.

\section{Acknowledgement}

I would like to acknowledge the patient and their relatives who had given consent and were cooperative to conduct this study. I must mention and acknowledge to the cardiology ward sisters, in charges, the family of department of cardiology and hospital authority; without their help this study would not have been possible.

\section{References}

1. A. Fanning. Tuberculosis: Extrapulmonary disease CMAJ 1999; 160:1597-603.

2. P.G. O' Neill, R. Rokey, S. Greenberg, et al. Resolution of ventricular tachycardia and endocardial 
tuberculoma following antituberculous therapy. Chest 1991;100(5) : 1467-9.

3. C. Wren, P.G. Stovin. Isolated interventricular septal tuberculoma causing complete heart block. Thorax 1982;37(2):149-50

4. S.G. Kinare, M.M. Deshmukh. Complete atrioventricular block due to myocardial tuberculosis. Arch Pathol 1969; 88:684-7

5. H. Krishnavani, G. Cherian. Right atrial tuberculoma : report of a case with complete recovery. Thorax 1984;39(7):550-1.

6. O.P. Kooper, E. Mascarenhas, M.M. Rananaware, et al. Tuberculoma of the heart. Report of 9 cases. Am Heart J 1973;86(3):334-40.

7. W.J. Rawls, W.H. Shufard, W.D et al. Right ventricular outflow tract obstruction produced by a myocardial abscess in a patient with tuberculosis. Am J Cardiol 1968;21:738-45.
8. R. Soyer, A. Brunet, B. Chevallier, et al. Tuberculosis aortic insufficiency: report of a case with successful surgical treatment. J Thorac Cardiovasc surg 1981;82:254-6

9. G. Bher, H.C. Palin, J.M. Temperley. Myocardial tuberculosis BMJ 1977:1:951

10. M.A. Dada, N.G. Lazarus, A.B. Kharsany, et al. Sudden death caused by myocardial tuberculosis: case report and review of the literature. Am J Forensic Med and Pathology 2000; 21(4):385-8.

11. L.T. Cooper Jr, G.J. Berry, R. Shabetai. Idiopathic giant cell myocarditis - natural history and treatment. Multicenter Giant Cell Myocarditis Study Group Investigators. N Engl J Med 1997; 336:1860-6

12. P.A. Vingola, K. Aonuma, P.S. Swaye, et al. Lymphocytic myocarditis presenting as unexplained ventricular arrhythmias: diagnosis with endomyocardial biopsy and response to immunosuppression. J Am Coll Cardiol 1984;4:8129. 